\title{
Marketing Olfativo en hoteles de lujo de Madrid centro
}

Olfactory Marketing in Luxury Hotels in Madrid Centre

\section{Andrea González Peña}

Grado en Publicidad y Relaciones Públicas con Dirección de Marketing y Gestión Comercial, ESIC Business \& Marketing School. Becaria,

Publicis Media Groupe y Netmentora Madrid andrealyceo@hotmail.com

\section{Dr. Juan M. González-Anleo}

Profesor ESIC Business \& Marketing School

juanmaria.gonzalezanleo@esic.edu
González Peña, A. y González-Anleo, J.M. (2020) Marketing Olfativo en hoteles de lujo de Madrid centro Revista Internacional de Investigación en Comunicación aDResearch ESIC. № 23 Vol 23

Segundo semestre, julio-diciembre 2020 • Págs. 90 a 107 https://doi.org/10.7263/adresic-023-05 
RESUMEN

\section{Clasificación JEL: \\ M31, M37 \\ Palabras clave:}

Marketing Olfativo,

Marketing Sensorial, hoteles de lujo,

olfato,

aroma
Objetivo: El objetivo de la presente investigación es descubrir la realidad existente en el sector hotelero de lujo de la zona centro de Madrid en lo concerniente a la implementación de estrategias y técnicas de Marketing Sensorial Olfativo a fin de revelar en qué medida se llevan a cabo tales estrategias en la actualidad, dónde, quién y con qué finalidad.

Diseño/metodología/enfoque: La investigación es de carácter cuantitativo y ha sido llevada a cabo mediante una encuesta tipo CAPI asistida por un Smartphone con un software que navega por el cuestionario. Dicha encuesta tuvo que ser completada por personal del hotel cualificado con los conocimientos necesarios para poder responder a las preguntas planteadas.

Resultados: Los resultados de la investigación validaron tres de las cinco hipótesis inicialmente planteadas para el estudio, por lo que se demostró que los hoteles de lujo de Madrid Centro utilizan tales estrategias para diferenciarse de la competencia y crear marca olfativa. Asimismo, se pone de relieve que un pequeño porcentaje de hoteles sigue sin usar dichas técnicas y que los niveles de satisfacción de los clientes se ven afectados por éstas positivamente entre otros resultados que serán expuestos más adelante.

Limitaciones/implicaciones: Debido a la responsabilidad y carga de trabajo que implican las funciones de las personas necesarias para responder la encuesta, no se obtuvieron respuestas de ciertos hoteles. De la planificación de veintiún hoteles inicialmente listados conseguimos catorce respuestas, todas ellas cara a cara.

Originalidad/contribución: Ha sido posible definir la realidad del Marketing Olfativo en el sector hotelero de lujo de Madrid Centro, así como las implicaciones que tiene y su contribución para crear una atmosfera agradable para los consumidores y garantizar su captación y fidelización a través de los aromas.

\section{ABSTRACT}

\section{JEL Classification: M31, M37}

\section{Key words:}

Olfactory Marketing, Sensory Marketing, Luxury Hotels, Sense of smell, Aroma
Purpose: The objective of this study is to discover the existing reality in the luxury hotel sector in the central area of Madrid concerning the implementation of Olfactory Sensory Marketing techniques and strategies with the aim of revealing to what extent, where, who by and with what end such strategies are carried out.

Design / Methodology / Approach: This is a quantitative study and has been carried out by means of an ICSC survey assisted by a Smartphone which navigates the questionnaire. The aforementioned survey had to be completed by hotel staff in possession of the required knowledge to be able to respond to the questions asked.

Results: The results of the research confirmed three of the five hypotheses initially proposed for the study, which demonstrated that the luxury hotels in Madrid Centre used such strategies to differentiate themselves from the competition and create an olfactory brand. Likewise, it highlights that a small percentage of hotels do not use said techniques as well as their positive effects on levels of customer satisfaction, along with other results which will be presented later.

Limitatios / Implications: Due to the responsibility and the workload which the functions of the people necessary to complete this survey incur, answers were not obtained from certain hotels. From the planned twenty-one hotels initially listed, fourteen replies were received, all of which were face-to-face.

Originality / Contribution: It has been possible to define the reality of Olfactory Marketing in the luxury hotel sector in Madrid Centre, along with the implications involved and its contribution to creating a pleasant atmosphere for consumers and guaranteeing their acquisition and retention by means of aromas. 


\section{Introducción}

Remontándonos en el tiempo puede constatarse que los aromas han tenido una profunda influencia en el comportamiento de los seres humanos desde su estado más primitivo. A través de ellos, los humanos primitivos eran capaces de diferenciar piezas de comida, intuir cuales eran peligrosas y cuales no o seleccionar de entre otros miembros la pareja reproductiva ideal. De hecho, existe una condición cuando una persona pierde la capacidad de percibir aromas llamada «anosmia» que provoca sentimientos de depresión y sensación de vacío. A pesar de todo esto, no obstante, el sentido del olfato ha sido el que menos atención ha recibido en la investigación científica relacionada con el marketing.

Sin embargo, hace relativamente poco, los profesionales del marketing comienzan a asumir la importancia del sentido olfativo para sus acciones y estrategias, especialmente para diferenciarse de sus competidores e incrementar los niveles de satisfacción y bienestar de sus consumidores pudiendo ser definido el Marketing Olfativo, de forma muy sucinta, como aquella rama específica del marketing encargada de estudiar cómo se utilizan los aromas para crear estados de ánimo, para promocionar productos o posicionar una marca (Krishna, 2010, pp. 57-67).

Pueden identificarse tres características fundamentales del aroma que ayudan a entender donde radica el origen de su importancia. En primer lugar, los aromas son difíciles de etiquetar pero fáciles de reconocer (Axel, 1995, pp. 154-159; Buck, 2004; pp. 116-119). Mientras un número bastante elevado de personas no es capaz de describir con palabras un aroma, sabe sin embargo perfectamente distinguir los olores inhalados con anterioridad. En segundo lugar, las preferencias por los aromas se originan en el aprendizaje. Varios estudios han demostrado que la predisposición para que determinados aromas sean o no del agrado de las personas viene dada por un aprendizaje continuo a lo largo del tiempo (Krishna, 2010, pp. 57-67; Herz, 2004, pp. 217-224). Cuando se tiene una experiencia positiva, se asocia el olor de esa experiencia a unas emociones positivas durante el resto de la vida sucediendo también lo contrario con experiencias negativas. Por último, si bien el aroma de un determinado elemento es procesado de forma lenta, éste persiste en el tiempo. El olfato es, en este sentido, el sentido más lento para percibir un estímulo, llegando el ser humano a tardar hasta 10 veces más en notar la presencia de un aroma que en reconocer una imagen (Goddard, 2005, pp. 79-86). Esto se debe a que la información de las neuronas olfativas tarda más tiempo en desplazarse hasta las neuronas del cerebro. A pesar de ello, no obstante, tiene una ventaja primordial, y es que el recuerdo marcado con una pista olfativa es mucho más persistente en la memoria.

Existen varias dimensiones para explicar cómo se forman las preferencias por los aromas. La primera es la hedónica, a través de la cual se determinan los aromas que resultan agradables y los que no. Al evaluar un aroma, la primera respuesta que viene en mente suele ser ésta, existiendo tres variables esenciales: la afabilidad, la familiaridad y la intensidad. Las dos primeras están estrechamente relacionadas y tienen connotaciones positivas; la intensidad, por el contrario, resulta más compleja, presentándose los registros en forma de U invertida, de tal forma que si el estimulo sobrepasa la capacidad media de percepción humana, el efecto producido puede llegar a ser el opuesto al deseado.

La segunda dimensión es el tipo de aprendizaje asociativo, cuya investigación se hace en referencia al condicionamiento clásico o pavloviano (Krishna, 2010, pp. 75-138). Esta segunda 
dimensión Implica respuestas automáticas y está basado en la vinculación entre un estímulo nuevo (aroma) con un reflejo ya existente (emoción) dada por la experiencia vivida. El aroma, por lo tanto, puede desencadenar un estado de ánimo vinculado a la primera vez que el individuo se expuso a dicho estímulo y de la misma forma darle un significado hedónico propio que determina la percepción del aroma.

El estudio de diferentes culturas ofrece amplia información que demuestra que el aprendizaje asociativo predomina ante la percepción hedónica de los aromas a la hora de formar preferencias, lo que implica que las personas deciden qué aromas prefieren con respecto al aprendizaje adquirido y no en función de si satisface o no la dimensión hedónica. Un buen ejemplo ilustrativo de este fenómeno fue el intento por parte de los militares de U.S. de crear una «bomba apestosa». Esta idea, más o menos acertada, se topó con un problema esencial: fue absolutamente imposible encontrar un aroma que fuese considerado desagradable de forma univoca por distintos grupos étnicos (Dilks, 1999, p. 599).

La tercera dimensión, por ultimo, son las propias bases biológicas de la relación aroma-emoción. La estructura cerebral y su neuroanatomía explican la relación única entre las emociones y la asociación con estímulos olfativos. Además de la posición privilegiada de cercanía entre el nervio olfativo, la amígdala (expresión de emociones y memoria emocional) (Cahill, 1995, pp. 295-296) y el hipocampo (memoria de corto a largo plazo) (Eichenbaum, 2001, pp. 199-207), la informa-

\section{Esquema 1. Aprendizaje Asociativo (Condicionamiento clásico)}

Los dos estímulos por separado:

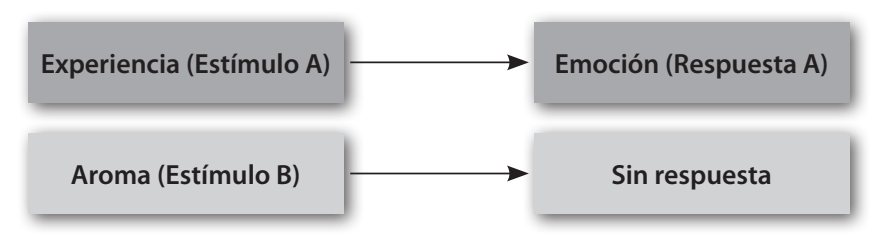

Los dos estímulos a la vez:

$1^{\text {a }}$ vez:

\section{Experiencia (Estímulo A) $\quad+\quad$ Aroma (Estímulo B) $\longrightarrow$ Emoción (Respuesta A)}

Sólo el Estímulo B tras haber tenido una primera toma de contacto con el Estímulo A:

$2^{\mathrm{a}}$ vez:

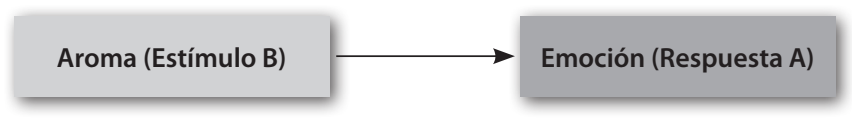

Fuente: Elaboración Propia 


\section{Esquema 2. El sentido del olfato, el cerebro y el cerebro límbico}

El sentido del olfato y el cerebro:

El cerebro límbico en profundidad:

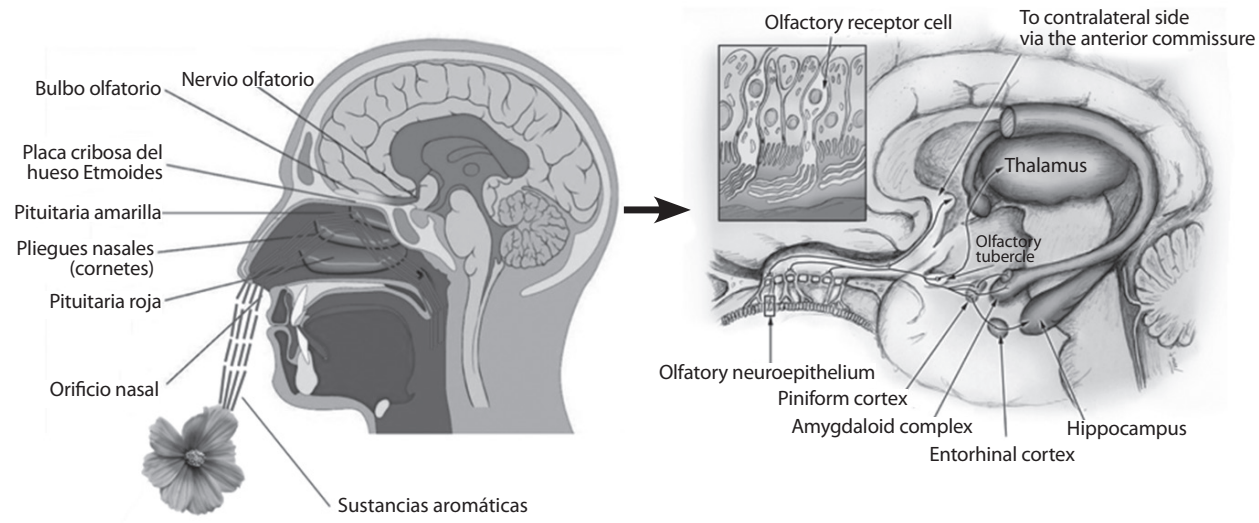

Fuente: Elaboración propia y otras fuentes de Google Sites

ción olfativa no tiene que pasar por el tálamo (almacén de información sensorial), sino que pasa de forma directa e inmediata a relacionarse con el complejo amígdala-hipocampo. Habiendo sido ampliamente demostrado que el aprendizaje asociativo de señales relativas a emociones específicas está mediado por la amígdala, ésta, junto al córtex olfativo, juegan un papel fundamental para el reforzamiento de estímulos aprendidos por aprendizaje asociativo.

Asimismo, puede señalarse que el sentido olfativo es un sentido químico, ya que percibe compuestos químicos en su entorno y puede ser efectivo a grandes distancias. El proceso es el siguiente:

1. El aroma en forma de moléculas de vapor hace su camino para llegar a las mucosidades de las fosas nasales y ahí se disuelven.

2. El olor es detectado por las neuronas receptoras del olfato situadas en las mucosidades.
3. Las mismas neuronas son responsables de transmitir la información a los bulbos olfatorios.

4. Los receptores sensoriales de estos bulbos se encargan de enviar mensajes a las estructuras del sistema límbico, pudiendo ser tanto a los centros primitivos del cerebro (neocorteza) como al centro «avanzado» en el que tienen lugar las modificaciones de los pensamientos conscientes.

Hay que destacar, en este contexto, que la relación neuroanatómica entre el olfato y la emoción está completamente vinculada a la neuroevolución. Las estructuras del sistema límbico (amígdala e hipocampo) se encontraban inicialmente ubicadas en el córtex olfativo, es decir, el aprendizaje emocional y asociativo provenía originalmente de una parte del cerebro que se dedicaba a procesar el sentido olfativo (Krishna, 2010 , pp. 75-134). Por ello se dice que la información de la emoción y del olfato son funcional- 
mente la misma, es decir, que las emociones, al igual que el olfato, conllevan respuestas que tienen una parte hedónica y otra basada en el aprendizaje asociativo y en las experiencias pasadas de las personas.

Esto llevó a entender a numerosos investigadores que los aromas pueden desencadenar estados de ánimo concretos en las personas. En este sentido, abundan los estudios que demuestran que los aromas agradables (perfume, aroma del pan recién horneado) mejoran el estado de ánimo e incluso alivian ciertas sensaciones de malestar (Villemure, 2003, pp. 101-108), así como los aromas desagradables que provocan un peor estado de ánimo (Knasko, 1992, pp. 27-35).

Otros estudios, además, demuestran que el estado de ánimo afecta a la cognición, es decir que estar de buen o mal humor puede afectar al comportamiento de los individuos y cambiar la percepción (Krishna, 2010, pp. 75-134). En términos generales, un estado de ánimo positivo provoca un incremento de la productividad y de la solidaridad, mientras que un estado de ánimo negativo causa un mayor estado de frustración y un efecto contrario en la productividad y en la sociabilidad (Baron, 1997, pp. 487-503).

Además de todo lo previamente abordado, hay que destacar también que la percepción del aroma depende del tipo de consumidor al que se dirija. La características sociodemográficas tienen influencia a la hora de percibir un aroma tal y como se demostró en un experimento realizado en París para la empresa Correos, cuyos resultados indicaron que entre dos grupos situados en zonas socioeconómicas contrarias las percepciones de los aromas fueron distintas. La cohorte generacional también tiene influencia en la percepción de los aromas (Maille, 2004, pp. 1-8). Así, Lindstrom (2005, pp. 67-71), demostró que los millenials son capaces de procesar información multisenso- rial de una forma mucho más efectiva que un consumidor medio perteneciente a generaciones anteriores con un cerebro más envejecido, como los baby boomers. Por otro lado, la influencia del hábito de consumo es importante, habiéndose demostrado (Ricaurte Bravo, 2013, pp. 1-73) que el aroma influye de forma significativa a los consumidores más «comedidos» para realizar gastos espontáneos.

Esta investigación surge, por lo tanto, de la necesidad de entender en mayor profundidad los procesos cerebrales que se producen a la hora de percibir e identificar aromas que posteriormente pueden suscitar emociones tanto positivas como negativas y, más concretamente, los efectos que pueden tener en los consumidores para que recuerden y prefieran una marca a otra, así como incluso llegar a cambiar sus comportamientos con respecto a la marca. En el caso concreto de los hoteles, para pasar más tiempo en ellos, para consumir más dentro de éstos, para crearles favoritismo y fidelizarles con la intención de que los recuerden y quieran volver a repetir la experiencia.

\section{Objetivos de la investigación}

En la actualidad, la tendencia de ofertar experiencias está creciendo exponencialmente, llegando a convertirse en un concepto esencial a la hora de captar la atención de los consumidores (Wikström, 2004, pp. 60-72). Así, las estrategias de marketing sensorial están cada vez más presentes en el sector de la hostelería de lujo (González Baeza; Ayala Montaño, 2019, pp. 1-32), y ello se debe a la necesidad de este sector por crear experiencias (mayormente hedónicas) de consumo para sus clientes debido a la interacción subjetiva que existe entre el personal de este tipo de negocios, el establecimiento en sí y los clientes (Pinheiro da Silva, 2010, pp. 330-345). El objetivo final de la implementación de estas estrate- 
gias es mejorar la estancia del cliente a través de las instalaciones del hotel gracias a la creación de sensaciones, emociones, pensamientos o cambios en el comportamiento de los consumidores aumentando, de esta forma, el recuerdo y la fidelización, así como diferenciarse de los competidores.

Dentro del Marketing Sensorial se ha escogido el Marketing Olfativo que orienta las acciones de marketing hacia la estimulación del sentido del olfato. Este es un sentido especialmente interesante por diferentes razones.

1. Es el sentido más primitivo y guarda, tal y como se ha visto, una estrecha relación con el sistema límbico, que es la estructura cerebral encargada de las emociones y la memoria (Cahill et al., 1995, pp. 295-296). Su ubicación es excelente porque se encuentra a dos sinapsis del nervio olfativo de la amígdala, encargada de expresar la emoción, y a tres sinapsis del nervio olfativo y el hipocampo, el cual transforma la memoria de corto a largo plazo (Krishna et al., 2010, pp. 57-67). Por ello, el olfato es fundamental en la percepción que se forma en la mente del consumidor frente a un producto o servicio.

2. El aroma de un producto o servicio permite al consumidor llevar a cabo una evaluación espontánea debido a su asociación con una experiencia a largo plazo (Morrin et al., 2000, pp. 157-165). Debido a ello, la memoria tiene un carácter experiencial y emocional asociando imágenes con sentimientos originados por recuerdos (Herz, 2000, pp. 34-39).

3. El sentido del olfato genera mayores niveles de motivación a nivel emocional en los individuos con respecto al resto de sentidos debido a su conexión con la memoria a largo plazo y la experiencia emocional del consu- midor (Beerli; Diaz; Martín-Santana, 2020, pp. 1-17).

La pregunta de origen planteada en nuestra investigación fue: ¿En qué medida los hoteles de lujo de Madrid implementan acciones de Marketing Olfativo, qué tipo de acciones y cómo influye esto en los resultados empresariales y en los niveles de satisfacción de los clientes?

Partiendo de esa base se planteó como objetivo general de la investigación recabar toda la información posible acerca de la implementación de estrategias de Marketing Olfativo en los hoteles de lujo de Madrid (capital) estableciendo, como objetivos específicos los que se detallan a continuación:

- Comprobar la presencia de acciones de Marketing Sensorial Olfativo en el sector de la hostelería de lujo en Madrid.

- Definir qué tipo de acciones de Marketing Olfativo se llevan a cabo.

- Encontrar en qué difieren estas estrategias dependiendo de la zona del hotel.

- Determinar si las acciones son llevadas a cabo por el propio hotel o por agencias independientes especializadas.

- Evaluar en qué medida estas acciones afectan a los resultados de la organización y cómo.

\section{Metodología empleada}

La presente investigación se ha llevado a cabo a partir de diferentes enfoques metodológicos. Inicialmente se elaboró un marco teórico originado en la lectura de diversas fuentes de expertos sobre Marketing Sensorial, poniendo el foco en el Marketing Sensorial Olfativo, el cual está orientado a estimular el sentido olfativo de los consumidores con objetivos diversos como la mejora de su estancia y el aumento del tiempo en ella, así como el incremento de su bienestar, 


\section{Mapa 1. Lista de los 14 hoteles que respondieron a la encuesta}

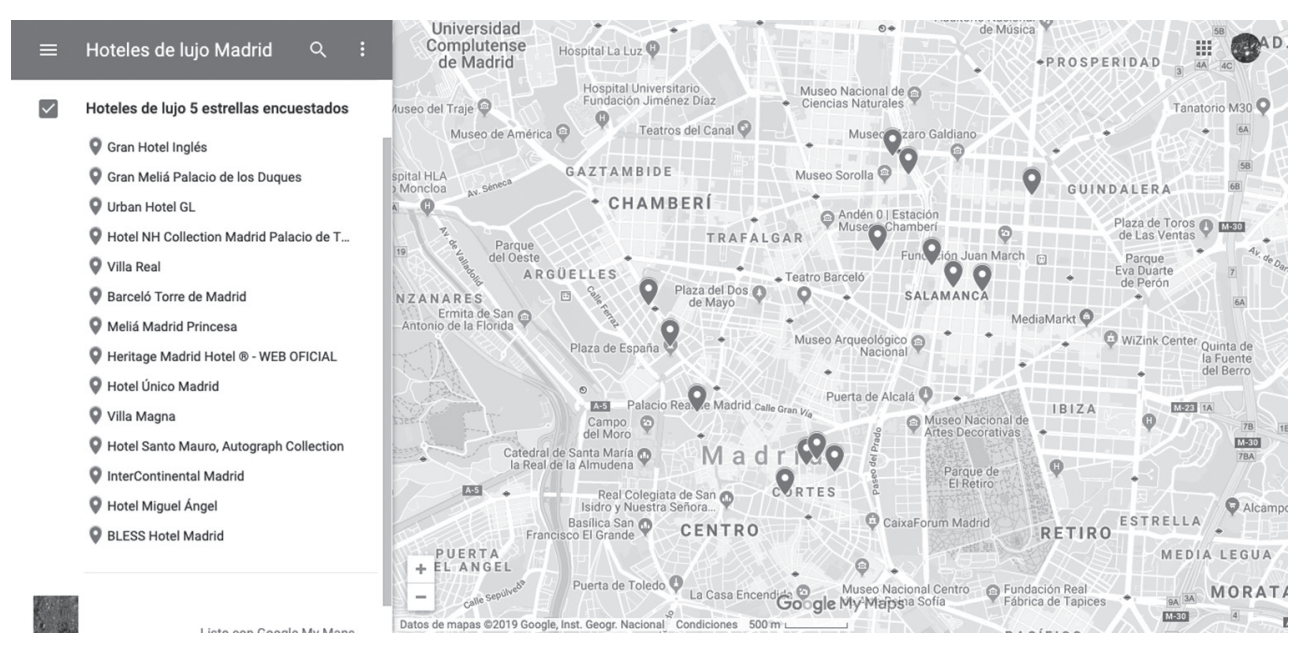

Fuente: Elaboración propia

su satisfacción y el sentimiento de pertenencia (fidelización).

La investigación puso especial interés en el sector de la hostelería de lujo, hoteles de 5 estrellas, dado que se trata de un mercado concreto al que las estrategias de marketing Olfativo pueden estar muy bien orientadas con el propósito final de crear una firma olfativa e identidad de marca. A través de esta investigación, por lo tanto, buscamos ahondar con mayor profundidad en el conocimiento de en que medida, cómo y con qué finalidad son implementadas estas estrategias en los hoteles de lujo del centro de Madrid, así como en la repercusión que puedan tener en los resultados de la organización.

En comparación con otros campos de estudio, el Marketing Sensorial no cuenta con un gran historial de investigaciones por lo que llevamos a cabo una investigación cuantitativa a través de una encuesta tipo CAPI que fue completada físicamente por un miembro del perso- nal cualificado de los distintos hoteles de lujo de Madrid visitados.

Es importante mencionar en este punto que el universo de los hoteles de 5 estrellas en la capital de Madrid es reducido. Se llevó a cabo una lista con todos los hoteles de lujo de la zona centro de Madrid (21 en total) y después, de forma aleatoria, se realizó una nueva lista con los hoteles a los que se debía visitar para que completaran la encuesta, consiguiendo que lo hiciesen 14 de ellos lo cual, teniendo en cuenta el reducido tamaño del universo, supone una muestra bastante representativa de la realidad.

\section{Limitaciones}

Por una parte, la muestra no es lo suficientemente grande para hacer extrapolaciones aún siendo representativa de la realidad. Además, teniendo en cuenta que la muestra inicial iba a ser de 21 hoteles, de lo cuales hubo 6 bajas, el tamaño de dicha tubo que verse reducido. 
Por otra parte, existe una limitación que apela a la satisfacción de los clientes, la cual no está completamente solventada por la investigación ya que habría hecho falta preguntarles a estos a través de otro cuestionario de satisfacción especialmente diseñado para este fin.

\section{Cuestionario}

El cuestionario se realizó de forma presencial a través de un Smartphone. La persona pertinente de cada hotel visitado rellenó dicho cuestionario en el momento, de modo a que también se pudieron solventar algunas dudas de manera oral durante el proceso.

Las personas encargadas de contestar dicho cuestionario tienen un perfil concreto que consta de un grado superior y/o universitario en las áreas de Marketing, Publicidad y Relaciones Públicas, Hostelería u otras ramas de estudio relacionadas conjunto a Títulos Superiores y/o Cursos y/o Másteres en Marketing Sensorial u Marketing Olfativo.

Las variables objeto de estudio de la investigación son cinco. La primera es el tipo de acciones desarrolladas y los sistemas implementados para llevarlas a cabo. La segunda variable es el tipo de acciones implementadas en función de las diferentes zonas del hotel. La tercera, el tipo de aromas utilizados por los hoteles, que se dividen en dos grandes grupos: genéricos y personalizados. La cuarta variable son los propios resultados de la empresa a distintos niveles (económico, volumen y satisfacción de los clientes). La quinta variable, por último, hace referencia a preguntas descriptivas de cada hotel.

Cada una de estas variables está definida por distintos tipos de preguntas, entre las que pueden distinguirse entre abiertas y cerradas. Las primeras son cortas y de desarrollo. Pero el mayor peso del cuestionario se lo llevan las preguntas cerradas, entre las cuales hay dicotomías, respuestas múltiples, respuestas mediante checkboxes y rating dentro de una escala de valores, como por ejemplo dando opciones desde «Totalmente de acuerdo» hasta «Totalmente en desacuerdo» o de forma más numérica siento el «1», por ejemplo, que los ingresos de la empresa han disminuido mucho y el «5» que

\section{Tabla 1. Clasificación de las preguntas del cuestionario}

\begin{tabular}{|c|c|c|}
\hline Variables objeto de estudio & $\begin{array}{l}\text { No } \\
\text { pregunta }\end{array}$ & Pregunta \\
\hline \multirow{5}{*}{$\begin{array}{l}\text { Tipos de acciones y } \\
\text { sistemas utilizados }\end{array}$} & 1 & ¿Están llevando a cabo acciones de Marketing Olfativo? \\
\hline & 2 & ¿Quién lleva a cabo estas acciones de Marketing Olfativo? \\
\hline & 3 & ¿Qué sistemas de aromatización utilizan actualmente? \\
\hline & 7 & $\begin{array}{l}\text { ¿Utilizan las mismas técnicas para destruir los malos olores que } \\
\text { para aromatizar espacios comunes? }\end{array}$ \\
\hline & 13 & $\begin{array}{l}\text { ¿Qué objetos de las habitaciones destinadas a los clientes } \\
\text { tienen un aroma añadido? }\end{array}$ \\
\hline \multirow{3}{*}{$\begin{array}{l}\text { Acciones en función de } \\
\text { la zona del hotel }\end{array}$} & 4 & ¿Emplean estrategias distintas en función de la zona del hotel? \\
\hline & 5 & ¿En qué zonas del hotel implementan estrategias olfativas? \\
\hline & 6 & $\begin{array}{l}\text { ¿Emplean diferentes aromas dependiendo de la zona } \\
\text { del hotel? }\end{array}$ \\
\hline
\end{tabular}


Tabla 1. Clasificación de las preguntas del cuestionario (continuación)

\begin{tabular}{|c|c|c|}
\hline Variables objeto de estudio & $\begin{array}{l}\text { No } \\
\text { pregunta }\end{array}$ & Pregunta \\
\hline \multirow{5}{*}{ Tipos de aromas utilizados } & 8 & ¿Utilizan aromas personalizados o aromas genéricos? \\
\hline & 9 & ¿Cómo hacen los aromas personalizados? \\
\hline & 10 & ¿Por qué hacen aromas personalizados? \\
\hline & 11 & Qué tipo de genérico usan? \\
\hline & 12 & ¿Prefieren los aromas genéricos o los personalizados? ¿Por qué? \\
\hline \multirow{3}{*}{$\begin{array}{l}\text { Influencia en los resultados } \\
\text { de la empresa }\end{array}$} & 15 & $\begin{array}{l}\text { «El nivel de satisfacción de los clientes ha aumentado desde } \\
\text { la implementación de dichas estrategias». Indique su nivel de } \\
\text { acuerdo o desacuerdo. }\end{array}$ \\
\hline & 16 & $\begin{array}{l}\text { Indique cómo han evolucionado los resultados económicos } \\
\text { de la empresa desde la implementación de estas estrategias } \\
\text { olfativas. }\end{array}$ \\
\hline & 17 & $\begin{array}{l}\text { ¿Cómo ha evolucionado el volumen de clientes desde la } \\
\text { implementación de estas acciones? }\end{array}$ \\
\hline \multirow{4}{*}{$\begin{array}{l}\text { Preguntas descriptivas } \\
\text { de cada hotel }\end{array}$} & 14 & ¿Desde hace cuánto están llevando a cabo dichas acciones? \\
\hline & 19 & ¿Cuál es el perfil de huésped que contrata sus servicios? \\
\hline & 20 & ¿Cuál es el nombre de su hotel? \\
\hline & 18 & $\begin{array}{l}\text { ¿Creen que los hoteles de la zona usan también éste tipo de } \\
\text { acciones orientadas al Marketing Olfativo? }\end{array}$ \\
\hline
\end{tabular}

Fuente: Elaboración propia

Tabla 2. Tipo de respuestas del cuestionario (Fuente: Elaboración propia)

\begin{tabular}{|c|l|l|}
\multirow{2}{*}{ No } & \multicolumn{2}{|c|}{ Pregunta } \\
\hline 1 & Dicotomía & \multicolumn{1}{|c|}{ Abierta } \\
\hline 2 & Múltiple & \\
\hline 3 & Checkboxes & \\
\hline 4 & Dicotomía & \\
\hline 5 & Checkboxes & \\
\hline 6 & Múltiple & \\
\hline 7 & Múltiple & \\
\hline 8 & Múltiple & \\
\hline 9 & & Larga \\
\hline 10 & Checkboxes & \\
\hline
\end{tabular}

\begin{tabular}{|c|c|c|}
\hline \multirow{2}{*}{$\begin{array}{l}\text { No } \\
\text { pregunta }\end{array}$} & \multicolumn{2}{|c|}{ Pregunta } \\
\hline & Cerrada & Abierta \\
\hline 11 & & Corta \\
\hline 12 & & Larga \\
\hline 13 & Checkboxes & \\
\hline 14 & Múltiple & \\
\hline 15 & Escala & \\
\hline 16 & Escala & \\
\hline 17 & Escala & \\
\hline 18 & Múltiple & \\
\hline 19 & Checkboxes & \\
\hline 20 & & Corta \\
\hline
\end{tabular}


han aumentado mucho. De este modo se hizo un cuestionario lo más variado posible e intuitivo.

\section{Antecedentes y resultados}

A pesar de que el Marketing Sensorial es un campo en el cual expertos como Krishna, Lindstrom y Herz han demostrado, a través de diferentes estudios y experimentos de diferente naturaleza, la eficacia de estas estrategias, aún son muchas las empresas que no tienen en cuenta la importancia de esta nueva forma de mercadeo, sobre todo si nos ceñimos al ámbito nacional.

Se escogió el mercado de los hoteles de lujo por ser un ámbito pertinente para la implementación de dichas estrategias y, además, como ya ha sido mencionado, por el amplio número de variables relacionadas con su implementación (González Baeza; Ayala Montaño, 2019, pp. 1-32).

Dichos hoteles utilizan diferentes técnicas para aromatizar sus espacios y la mayoría se dirige a mejorar el ambiente ya que puede ejercer una influencia importante en la toma de decisiones de los clientes (Rivera; Méndez, 2020, pp. 51-61), en parte debido a que el servicio necesita la implicación del consumidor y el personal de forma simultánea, donde reside la importancia de las condiciones ambientales idóneas durante la prestación y la consumición del servicios creando una experiencia positiva para el cliente (Wikström, 2004, pp. 60-72). Es fundamental la implementación de estas estrategias en las cadenas de hoteles de lujo ya que a través de estas es más probable que los consumidores repitan su estancia en el hotel si conservan un recuerdo positivo de la primera experiencia a pesar de estar en otra ciudad (Carolina Sarmiento, 2014, pp. 1-87). De hecho, esto se confirma en los resultados que muestran que los hoteles utilizan estas técnicas olfativas con dos objetivos: el $100 \%$ de los encuestados afirma que es por la creación de una firma olfativa que les haga únicos y un $75 \%$ por el aumento de la satisfacción global de los clientes y su bienestar (pregunta $\mathrm{n}^{\circ} 10$ ). Además, hay estudios que demuestran que a través de la implementación de estas acciones mejora la percepción de los cliente con relación a la calidad del propio servicio del hotel (García Mayorga, 2019, pp. 6-14).

Mehrabian y Russell fueron, en 1974, los primeros investigadores en interesarse por la influencia que puede ejercer el ambiente y su acondicionamiento al comportamiento de los consumidores. Según ellos, las posibles respuestas de los consumidores ante un ambiente determinado pueden ser de aproximación (si la atmosfera es positiva) o de alejamiento (si la atmosfera es negativa) y éstos derivan de emociones concretas de los consumidores (Bitner, 1992, pp. 57-71). Crear una atmosfera positiva implica que el consumidor quiera pasar más tiempo en el establecimiento por lo que habrá más posibilidades de que inviertan en él, adquieran más servicios y productos y que además quieran repetir con la misma marca hotelera. Según los resultados un 60 \% de los encuestados afirma que el nivel de satisfacción de los clientes aumentó desde la implementación de dichas estrategias olfativas (pregunta $n^{\circ} 15$ ), con lo cual es verdad que crear una atmosfera positiva permite que los clientes disfruten más de su estancia y les resulte más placentera. Además, los encuestados afirmaron que los propios clientes comentan positivamente y preguntan en ocasiones por el aroma de las estancias para poder adquirirlo y a veces incluso los propios hoteles venden su fragancia como en el caso de Vincci Hoteles. Por otra parte, el 90 \% de las respuestas indica que a nivel de ingresos y de volumen de clientes no hubo ningún cambio tras la implementación de dichas acciones ya que los resultados se mantuvieron estables durante los años 
siguientes tras la puesta en práctica de dichas estrategias (pregunta $n^{\circ} 16$ y 17) por lo que no resultan significativas desde un punto de vista económico sino desde un punto de vista emocional, es decir, que los hoteles buscan una vinculación emocional con los clientes para que estos se sientan satisfechos, recuerden la marca y repitan la experiencia.

Hay algunas empresas que invierten en el desarrollo de un odotipo (firma olfativa) con la finalidad de ser reconocibles por el propio aroma desarrollado, más allá del logotipo o de sus productos y servicios. Es una apuesta cada vez más necesaria para las empresas que quieren explorar nuevas formas de comunicación. Los resultados indican que el $64 \%$ de los encuestados diseñan un odotipo u aroma personalizado para su hotel, otro $9 \%$ utiliza ambos, dependiendo de las zonas del hotel, por ejemplo, en las zonas comunes donde suele haber más afluencia de personas como son el vestíbulo y los servicios utilizan el odotipo y en zonas con menor afluencia aromas genéricos (preguntas n4, 5 y 8). El otro $30 \%$ de respuestas indica su inclinación hacia el uso de aromas exclusivamente genéricos. Entre ellos, los más utilizados son el bambú, flor di acqua, jazmín y orquídeas (pregunta $\mathrm{n}^{\circ} 11$ ).

Empresas como Abercombie, Disney o Rolls Royce llevan mucho tiempo haciendo uso de este tipo de técnicas con resultados bastante positivos a pesar de que siempre tienen que llevar controles muy estrictos por las posibles alergias que los químicos puedan causar en los individuos.

Es importante destacar que el aspecto más importante para desarrollar un aroma es la congruencia (Beerli; Diaz; Martín-Santana, 2020, pp. 1-17). Este concepto hace referencia a la importancia de la relación entre las características de un producto, el espacio, la marca y el aroma que se le da para potenciarlas. Dicho de otra forma, con- siste en la comunicación que realizamos por medio del olfato sea coherente con el resto de comunicaciones corporativas (Maille, 2004, pp. 1-8).

Existen distintas clasificaciones para los aromas en función, por ejemplo, de la propia cualidad aromática del olor y sus efectos directos en el estado físico y anímico del sujeto, aunque, como ya ha ido apuntando, existen muchos más factores más allá del propio aroma como la experiencia vivida y la emoción asociada previamente con el aroma en cuestión. La clasificación de aromas que se puede llevar a cabo y la que más nos interesa en este trabajo, es la de aromas identificativos, asociativos y reproductivos (González Baeza; Ayala Montaño, 2019, pp. 34-39)

- Lo aromas identificativos son conocidos también como odotipos, logos olfativos o firmas olfativas que buscan asociar un aroma determinado con la memoria a fin de identificar la marca y hacerla más memorable debido a la estrecha relación del olfato con la memoria. En nuestro estudio, el 100 \% de los encuestados manifiesta su preferencia por esta categoría debido a que los clientes tienden a identificar y diferenciar con mayor facilidad a la marca (pregunta $\mathrm{n}^{\circ} 12$ ).

- Los aromas asociativos, por su lado, son aquellos que tienen una asociación subjetiva con ciertos sectores comerciales, como el olor a chucherías en las tiendas de niños.

- Los aromas reproductivos, por último, tienen como finalidad potenciar el aroma natural de un producto para incrementar su consumo, relacionando directamente el aroma con una buena impresión y sensación de calidad por parte del consumidor.

Los resultados muestran que los hoteles de lujo implementan estrategias de Marketing Olfativo para diferenciarse de sus competidores, atraer 
a clientes potenciales y fidelizar a clientes actuales. Entre las razones que nos dan los encuestados, crear marca olfativa y diferenciarse de la competencia son las que más peso tienen (preguntas $\left.\mathrm{n}^{\circ} 10-12\right)$. Aunque no todos los hoteles de esta categoría llevan a cabo estas acciones, ya que de 14 hoteles encuestados, 3 de ellos están en esta situación por razones de política de empresa en cuyo caso únicamente hacen uso de la máquina de ozono para eliminar los malos olores y de aerosoles para neutralizar el olor a cerrado que dejan estas maquinas, pero sin ninguna intencionalidad de aromatizar las estancias (pregunta $n^{\circ} 1$ y 7 ).

Existen distintitas entidades y agencias dedicadas al Marketing Olfativo, según Martin Resino Jamarillo (2018) a nivel nacional tenemos Aromatizame, Pressentia, Sensology, The Sensory Lab, Dejá_vù Brands, Ambifresh, Pro-Air, Grupo Hueles, Aromas \& Co, Best Aroma y Akewele. Durante la investigación una de las principales cuestiones era saber si son los propios hoteles quienes llevan a cabo el diseño de estos aromas o si lo hacen a través de terceros o a través de la contratación de servicios de agencias. Según los resultados obtenidos, el $46 \%$ de estas estrategias son implementadas por los propios hoteles, el $36 \%$ a través de colaboración conjunta de un especialista y el propio hotel, y el $18 \%$ restante estaba bajo el control de una agencia externa especializada (pregunta $n^{\circ} 2$ ). En los casos que existe una colaboración conjunta, por lo general se debe a que los hoteles utilizan sus propios medios para implementar dichas acciones, es decir, que adquieren y utilizan los sistemas de aromatización que creen convenientes, y los terceros, ya sea agencia o especialista les diseña y suministra el aroma personalizado por el que han pagado.

Los métodos de difusión que elijan los hoteles deben tener en cuenta no sólo el presupuesto del que parten sino también las dimensiones del área

\section{Gráfico 1. Razones para la creación de odotipo}

Crear firma olfativa (47\%)

Aumentar notoriedad de marca (12\%)

Estancias concretas (6\%)

Aumentar bienestar de los clientes (35\%)

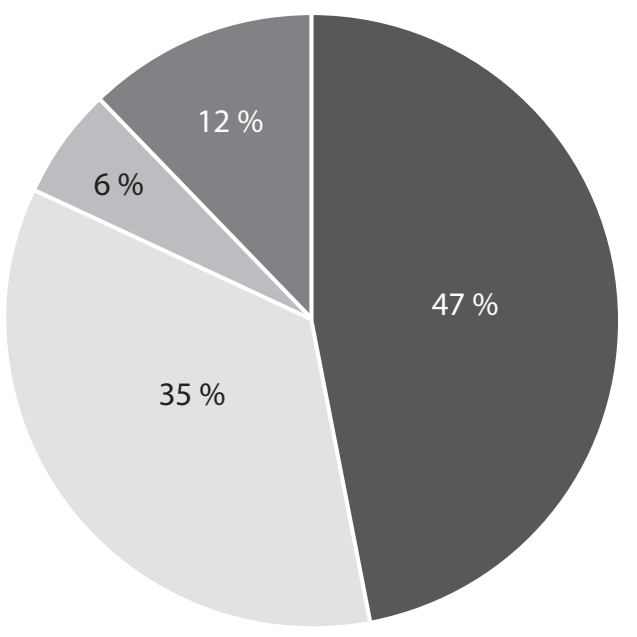




\section{Gráfico 2. Presencia de los sistemas de aromatización}

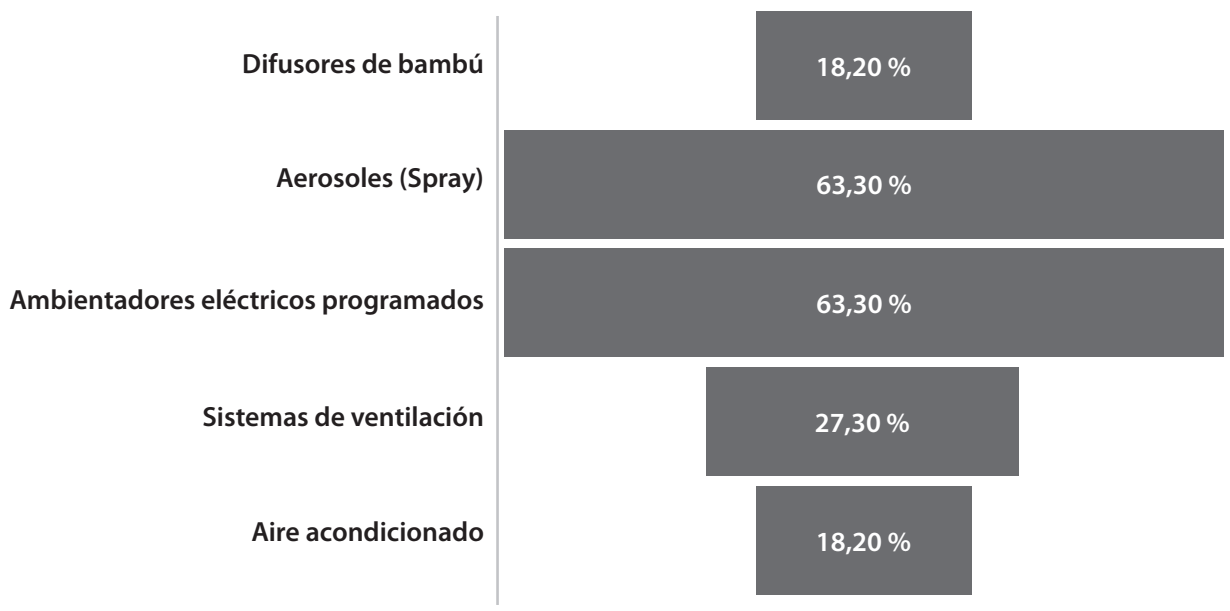

Fuente: Elaboración propia

que se pretende aromatizar (Sarmiento, 2012). Se divide en tres categorías, sistemas para espacios pequeños que vienen a ser velas o difusores, sistemas para espacios grandes que suele ser a través de los sistemas de ventilación y del aire acondicionado y por último los sistemas para puntos intermedios que suelen ser aerosoles o spray. Se quiso saber cuales eran los sistemas de aromatización más utilizados y a través de la encuesta y se descubrió que había dos sistemas que despuntaban sobre los demás: los aerosoles de spray y los ambientadores eléctricos programados. Seguidos irían los sistemas de ventilación, el aire acondicionado y, finalmente, los difusores de bambú, siendo los menos utilizados (pregunta ${ }^{\circ} 3$ ). Según se ha comprobado, los hoteles de esta categoría tienen entre uno y tres tipos distintos de sistemas de aromatización. Los hoteles que suelen poseer un solo tipo de sistema se decantan por los ambientadores eléctricos pro- gramados aunque no es extraño encontrarlo complementado por otros sistemas, entre los cuales el aerosol es el más común.

\section{Conclusiones}

Este proyecto de investigación se ha centrado en estudiar la necesidad de las empresas en la actualidad por implementar nuevas estrategias de Marketing, en concreto, se ha puesto la mira en investigar el campo del Marketing Sensorial Olfativo. Durante la búsqueda de un marco teórico que permitiese comprender cómo funciona el sentido del olfato y cómo afecta éste a los consumidores, se descubrió que la necesidad de la utilización de este tipo de estrategias proviene de la saturación que existe actualmente en el mercado de estrategias de Marketing Tradicional y la necesidad de innovar a la hora de atraer a los consumidores a través de sus sentidos como señala Kotler (1993, pp. 48-65). 
Analizando los resultados obtenidos se ha podido observar la presencia de acciones de Marketing Olfativo en hoteles de lujo (5 estrellas) del centro de Madrid, al no ser un universo muy grande, la muestra es significativamente representativa. Por lo menos un $79 \%$ de los hoteles encuestados llevan a cabo estas estrategias, el porcentaje restante no lo hace por políticas de empresa y porque ya lleva a cabo la eliminación de malos olores con la ayuda de la máquina de ozono. Estas acciones son llevadas a cabo por el propio hotel, agencias especializadas o un trabajo en conjunto de estos dos últimos. Según se ha visto, los sistemas de aromatización más utilizados son los aerosoles y los ambientadores eléctricos pero esto va a depender del tamaño de las estancias que se quieran aromatizar, por lo que es normal encontrar diferentes sistemas de aromatización en función de las estancias aromatizadas. Las zonas comunes y con mayor afluencia de personas como el vestíbulo y los servicios son aquellas que por lo general todos los hoteles coinciden que deben de ser aromatizadas.

En algunos casos se han encontrado hoteles que utilizan distintos aromas dependiendo de la zona del hotel que por lo general usan aromas genéricos o personalizados. El 64 \% de los hoteles usan aromas personalizados y el $100 \%$ pre- fiere los aromas personalizados, eso se debe a que buscan crear una firma olfativa u odotipo a través del cual el cliente les reconozca y les recuerde con facilidad, asimismo lo hacen para aumentar la propia satisfacción de los clientes (Beerli; Diaz; Martín-Santana, 2020, pp. 1-17) ya que si estos vinculan una experiencia positiva en el hotel a dicho aroma, lo más probable es que la próxima vez que perciban el mismo aroma les provoque emociones positivas ligadas al recuerdo positivo vinculado y quiera repetir la experiencia.

Por otro lado, los resultados de nuestro estudio muestran con claridad que en lo que se refiere al nivel económico y de volumen de clientes, la implementación de estas estrategias de Marketing Olfativo no tiene ningún efecto, siendo sin embargo de importancia a la hora de aumentar el nivel de satisfacción y bienestar de los clientes.

En conclusión, la implementación de estrategias de Marketing Olfativo tiene múltiples beneficios para el sector del ocio, especialmente para la hostelería de lujo, dada su capacidad para incrementar el bienestar de sus clientes, así como para la creación de una marca diferenciada y fácilmente reconocible que incremente su notoriedad, «memorabilidad»y por lo tanto el nivel de repetición de los clientes y su fidelidad hacia la marca. 


\section{Bibliografía}

Axel, R. (1995). The molecular logic of smell. Scientific American 273, 154-159.

Baron, R. A. (1997). The sweet smell of helping: Effects of pleasant ambient fragrance on prosocial behavior in shopping malls. Personality and Social Psychology Bulletin, 23, 487-503.

Beerli, A.; Diaz, G.; Martín-Santana, D.J. (2020). Satisfaction, image, and loyalty can be enhanced with congruent olfactory treatments: the acid test of optician franchise stores in shopping centres and on the high street, Journal of Strategic Marketing, 1-17.

Bitner, M. J. (1992). Servicescape: the impact of physical surroundings on customer and Employees. Journal of Marketing, 56 (2), 57-71. Disponible en: https://www.ida.liu. se/ steho87/und/htdd01/9208310667.pdf

Buck, (2004). The search for odorant receptors. PubMed Central (PMC), 116, 116-119.

Sarmiento, C. (2014). Marketing Olfativo en la línea hotelera de lujo, una estrategia de creación de identidad de marca, Universidad Espíritu Santo, 1-87. Disponible en: https://linkinghub.elsevier.com/retrieve/pii/S0092867404000510

Cahill, L. Babinsky, R., Markowitsch, H.J., McGaugh, J. L. (1995). Amygdala and emotional memory. Nature, 337, 295-296. Disponible en: https://www.nature.com/articles $1377295 \mathrm{a} 0$

Dilks, D. D., Dalton, P., Beauchamp, G. K. (1999). Crosscultural variation in responses to malodors. Chemical Sense, 24,599 .

Eichenbaum, H. (2001). The hippocampus and declarative memory: Cognitive mechanisms and neural codes. Behavioral Brain Research, 127, 199-207. Disponible en: https://www. sciencedirect.com/science/article/pii/S01664328 01003655?via\%3Dihub

García Mayorga, D. C. (2019). Marketing sensorial para mejorar la percepción de la calidad del servicio en el Hotel El Libertador de la ciudad de Riobamba. Escuela Superior Politécnica de Chimborazo, 6-14. Disponible en: http://dspace. espoch.edu.ec/handle/123456789/13697

Goddard, L., Pring, L., \& Felmingham, N. (2005) The effects of cue modality on the quality of personal memories retrieved. Taylor \& Francis Group, 13 (1), 79-86. Disponible en:https://www.tandfonline.com/doi/abs/10.1080/ 09658210344000594
González Baeza, M.; Ayala Montaño, K. F. (2019). El Marketing Experiencial y su importancia en el Mundo Hotelero, Facultad de Economía, Empresa y Turismo, 1-32.

Herz, R. S. (2000). Scents of time. The Sciences, 34-39. Disponible en: https://riull.ull.es/xmlui/handle/915/15023

Herz, R. S. (2004). A comparison of autobiographical memories triggered by olfactory, visual and auditory stimuli. Chemical Senses (29), 217-224.

Knasko, S. C. (1992). Ambient odor's effect on creativity, mood and perceived health. Chemical Senses (17) 27-35.

Kotler. (1993). Atmospherics as a marketing tool. Journal of Retailing, 49 (4), 48-65.

Krishna, A, Lwin, M. O. \& Morrin, M. (2010). Product scent and memory. The Journal of Consumer Research, 37 (1), 57-67. Disponible en: https://doi.org/10.1086/649909

Krishna, A. (2010). Sensory Marketing: Research on the Sensuality of Products, 75-134, 364-366. London. Routledge.

M. Lindstrom. (2005). Brand Sense: Build powerful brands through touch, taste, smell, sight and sound. The Free Press. London.

M.J Ricaurte Bravo. (2013). Marketing Sensorial: El efecto de los Estímulos Olfativos y Visuales en el Punto de Venta Universidad San Francisco de Quito, 1-73. Disponible en: http://repositorio.usfq.edu.ec/handle/23000/2762

Maille, V. (2004). La incidencia del estímulo olfativo en el punto de venta: el ejemplo de Correos, Percepnet, 1-8. Disponible en: http://www.percepnet.com/cien05_04.htm

Mehrabian, A., Russell, J.A. (1974). An approach to environmental psychology. MIT Press, 135-200.

Morrin, M., Ratneshwar, S. (2000). The impact of ambient scent on evaluation, attention, and memory for familiar and unfamiliar brands. Journal of Business Research, (49), 157 165. Disponible en: https://linkinghub.elsevier.com/retrieve/pii/S0148296399000065

Perdomo Rivera, W. F., Camelo Méndez, W. Y. (2020). Efectos de las variables ambientales sobre la decisión de compra del consumidor de: Fruti helado. Fundación universitaria Los Libertadores, 51-61. Disponible en: http://hdl.handle.net/ $11371 / 2658$

Pinheiro da Silva, J., Vasconcelos Pereira Leite, Y. (2010). Hospitalidad y ambiente en los hoteles. Su influencia en el comportamiento del consumidor. Estudios y Perspectivas en Turismos, 19 (3), 330-345. Disponible en: https://dialnet. unirioja.es/servlet/articulo? codigo $=3351500$ 
Villemure, C., Slotnick, B. M., Bushnell, M. C. (2003). Effects of odors on pain perception: deciphering the roles of emotion and attention. Pain, 106 (1-2), 101-108. Disponible en: https://doi.org/10.1016/s0304-3959(03)00297-5
Wikström, S. (2004). When products and service are not enough. ECR Journal International Commerce Review, 4 (2), 60-72.

\section{Anexo:}

1. ¿Están llevando a cabo acciones de Marketing Olfativo?

2. ¿Quién lleva a cabo estas acciones de Marketing Olfativo?

3. ¿Qué sistemas de aromatización utilizan actualmente?

4. ¿Emplean estrategias distintas en función de la zona del hotel?

5. ¿En qué zonas del hotel implementan estrategias olfativas?

6. ¿Emplean diferentes aromas dependiendo de la zona del hotel?

7. ¿Utilizan las mismas técnicas para destruir los malos olores que para aromatizar espacios comunes?

8. ¿Utilizan aromas personalizados o aromas genéricos?

9. ¿Cómo hacen los aromas personalizados?

10. ¿Por qué hacen aromas personalizados?

11. ¿Qué tipo de genérico usan?
12. ¿Prefieren los aromas genéricos o los personalizados? ¿Por qué?

13. ¿Qué objetos de las habitaciones destinadas a los clientes tienen un aroma añadido?

14. Desde hace cuánto están llevando a cabo dichas acciones?

15. «El nivel de satisfacción de los clientes ha aumentado desde la implementación de dichas estrategias" Indique su nivel de acuerdo o desacuerdo.

16. Indique cómo han evolucionado los resultados económicos de la empresa desde la implementación de estas estrategias olfativas.

17. ¿Cómo ha evolucionado el volumen de clientes desde la implementación de estas acciones?

18. ¿Creen que los hoteles de la zona usan también éste tipo de acciones orientadas al Marketing Olfativo?

19. ¿Cuál es el perfil de huésped que contrata sus servicios?

20. ¿Cuál es el nombre de su hotel? 
